\title{
Evolution and dispersal of three closely related macaque species, Macaca mulatta, M. cyclopis, and M. fuscata, in the eastern Asia
}

\author{
Jui-Hua Chu ${ }^{\mathrm{a}}$, Yao-Sung Lin ${ }^{\mathrm{a}}, \mathrm{Hai}-\mathrm{Yin} \mathrm{Wu}^{\mathrm{b}, *}$ \\ ${ }^{a}$ Institute of Ecology and Evolutionary Biology, National Taiwan University, Taipei 106, Taiwan, ROC \\ ${ }^{\mathrm{b}}$ Institute of Natural Resources, National Dong Hwa University, Hualien 974, Taiwan, ROC \\ Received 7 April 2006; revised 15 November 2006; accepted 16 November 2006 \\ Available online 5 December 2006
}

\begin{abstract}
Macaca mulatta, M. cyclopis and M. fuscata are three closely related species in the fascicularis species group. M. mulatta is wide-spread in Asia, while M. cyclopis and M. fuscata are restricted to Taiwan and Japan, respectively. Both M. cyclopis and M. fuscata are thought to be derived from ancient 'mulatta' populations in the eastern Asia. In this study, we analyzed sequences of mitochondrial DNA control region to provide genetic evidence for the evolution and dispersal scenario of the three species proposed by Fooden and Albrecht [Fooden, J., Albrecht, G.H. 1999. Tail-length evolution in fascicularis-group macaques (Cercopithecidae: Macaca). Int. J. Primatol. 20, 431440]. Our results indicated that several localities in the southern China and Vietnam harbored multiple divergent mtDNA lineages that may not have evolved sympatrically. These divergent mtDNA lineages may have originated from different ancient northern populations that retreated into southern localities during glacial periods. However, the age of the southward retreat and the northward recolonization may be dated back to a more ancient past during late middle Pleistocene (0.12-0.18 mya) instead of during the LGM (0.018 mya). Times of gene divergence between $M$. mulatta and the two island species, estimated by mean nucleotide difference, suggest the ancestral populations colonized Taiwan and Japan around 0.38-0.44 mya. In addition, a more recent age of mulatta-cyclopis-fuscata population divergence (when ancient populations were isolated), estimated to be 0.17 mya by net nucleotide divergence, is suggested.
\end{abstract}

(C) 2006 Elsevier Inc. All rights reserved.

Keywords: Macaca mulatta; M. cyclopis; M. fuscata; mtDNA control region; Phylogeography; Eastern Asia

\section{Introduction}

The 19 extant species of the genus Macaca (BrandonJones et al., 2004) have been organized into three to six species groups according to morphological or genetic differences (Delson, 1980; Fooden, 1976; Fooden and Lanyon, 1989; Groves, 2001; Hayasaka et al., 1996; Li and Zhang, 2005; Zhang and Shi, 1993). Among these species groups, the evolutionary relationship of the fascicularis species group, composed of Macaca fascicularis, M. mulatta, M. cyclopis and M. fuscata, is of particular interest because paraphyly has been observed from genetic data (Hayasaka

\footnotetext{
* Corresponding author. Fax: +88638633260.

E-mail address: hywu@mail.ndhu.edu.tw (H.-Y.Wu).
}

et al., 1996; Melnick et al., 1993; Morales and Melnick, 1998; Tosi et al., 2000, 2002, 2003). Based on maternally, paternally and bi-parentally inherited gene sequences, evolutionary relationship of the fascicularis species group has been described by Melnick and colleagues (Melnick et al., 1993; Morales and Melnick, 1998; Tosi et al., 2000, 2002, 2003). According to their studies, M. fascicularis is the ancestral form among the four species. M. mulatta may have originated from a fascicularis-like ancestor 2.5 million years ago (mya) and became widely distributed within a relatively short period. The rapid expansion was followed by mitochondrial differentiation between the eastern and western parts of the species range (about 0.75 mya). After the intraspecific divergence, populations of eastern M. mulatta (China-Burma) colonized Taiwan and Japan during glacial periods when land bridges were available. Mitochondrial 
differentiation between M. mulatta and M. cyclopis or $M$. fuscata was estimated to be 0.25 and 0.50 mya, respectively.

Female philopatry of Macaca species maintains a geographic structuring of the maternally inherited mitochondrial DNA (mtDNA) that reflects patterns of cladogenic events (Melnick et al., 1993). It explains the observation that mtDNA haplotypes of eastern M. mulatta (those from China) are more similar to haplotypes of the two insular species (M. cyclopis and M. fuscata) than to haplotypes of western $M$. mulatta (those from India-Pakistan), or mtDNA paraphyly of M. mulatta (Melnick et al., 1993; Morales and Melnick, 1998). However, such pattern was not detected in Y-chromosome (Tosi et al., 2000). This can be attributed to male-mediated gene flow between eastern and western M. mulatta that homogenized nuclear genetic variation across geographic range (Tosi et al., 2002, 2003). Moreover, a southward introgression by male $M$. mulatta to populations of $M$. fascicularis generated a paraphyletic Y-chromosome pattern in M. fascicularis (Tosi et al., 2002, 2003).

According to Allen's rule, size of extremities such as ears and tails of mammals increases from colder to warmer climates in the same or closely related species, especially for those inhabiting a large latitudinal range. The composite distribution range of members in the fascicularis species group encompasses a wide latitudinal zone from $10^{\circ} \mathrm{S}$ to $40^{\circ} \mathrm{N}$. The relative tail length (RTL, tail length/head and body length) in the species group generally decreases with increasing latitudes just as predicted by Allen's rule (Fooden, 1997). However, the correlation does not apply to $M$. mulatta inhabiting the $15^{\circ} \mathrm{N}-25^{\circ} \mathrm{N}$ latitudinal zone, i.e. the southern populations (Fooden and Albrecht, 1999). RTL of extant southern mulatta populations is less than that of $M$. cyclopis living in similar latitudes, but is similar to that of the conspecific northern populations. Fooden and Albrecht (1999) also in Fooden (2000) suggested that modern southern mulatta populations did not originate within their present range; instead, they may be descendents of the populations retreating from farther north. They proposed a hypothetical scenario for the evolution and dispersal of $M$. mulatta based on variation of RTL in the fascicularis species group as well as the evolutionary history of the mulatta group (including M. mulatta, M. cyclopis and M. fuscata), which will be briefly described in the following.

By the observed trend between latitude and RTL in the fascicularis species group, the ancient mulatta populations originally inhabited between $15^{\circ} \mathrm{N}$ and $25^{\circ} \mathrm{N}$ were probably more similar to $M$. cyclopis in RTL (mean $\mathrm{RTL}=0.80$, the long-tailed populations), while those inhabiting farther north were more similar to $M$. fuscata in RTL (mean $\mathrm{RTL}=0.15$ at $40^{\circ} \mathrm{N}$, the short-tailed populations). The latitudinal gradient in RTL was formed gradually during northward population expansion at least 0.04 mya. At this stage, the ancient mulatta populations colonized Taiwan and Japan when dispersing routes were available. Extant M. fuscata and M. cyclopis are two relictual populations descended from the ancient short-tailed populations and long-tailed populations, respectively. During the last glacial maximum (LGM, about 0.018 mya), when climatic deterioration compressed suitable habitats southward, the shorttailed populations (mean RTL $<0.50$ ) retreated from north in the eastern Asia and replaced the long-tailed populations. During the southward range shift, a west-east gradient of decreasing RTL had been established (mean $\mathrm{RTL}=0.45$ at $100^{\circ} \mathrm{E}$, mean $\mathrm{RTL}=0.30$ at $120^{\circ} \mathrm{E}$ ). However, the factors responsible for the gradient are not clear. Subsequent to the LGM, i.e. during the Holocene expansion, both the eastern and western populations of $\mathrm{M}$. $\mathrm{mul}$ atta dispersed northward.

If the southward dispersal of M. mulatta in the eastern Asia occurred during the LGM as described by Fooden and Albrecht (1999), the phylogenetic relationship of the extant local populations may not correspond to geographic affinity and the dispersal pattern may be hard to trace. Furthermore, dispersal pattern of M. mulatta during Holocene expansion (about 0.01 mya) should be apparent from the geographic distribution of closely related mtDNA haplotypes. However, the low divergent level of mitochondrial ribosomal RNA genes that Morales and Melnick (1998) used may not be sensitive enough to reveal recent differentiation. A more polymorphic mtDNA marker is needed.

Mitochondrial DNA polymorphism is commonly used to reveal phylogenetic and phylogeographic relationships between populations of a species or closely related species (Avise et al., 1987). The control region (CR), a unique noncoding nucleotide sequence, is the most variable portion of mtDNA in mammals (Saccone et al., 1993). The sedentary nature of female macaques and the maternal inheritance of mtDNA make the molecule an ideal tool to elucidate population history (Melnick et al., 1993). Given the scenario hypothesized by Fooden and Albrecht (1999), objectives in this study are: (1) to find genetic evidence that supports the southward retreat in M. mulatta; (2) to trace the possible dispersal patterns of northward range expansion of M. mulatta; and (3) to estimate the time of divergent events of ancestral M. cyclopis and M. fuscata.

\section{Materials and methods}

\subsection{Sample collection}

In this study, mtDNA CR sequences of the four species in the fascicularis species group were analyzed. Samples of 148 M. cyclopis (107 fecal, 32 hair and 9 tissue samples) collected from five regional populations in Taiwan (GenBank accession nos. AY878873-AY878925 and DQ143984DQ143987) and 5 M. fascicularis of unknown origin (GenBank accession nos. AY884307-AY884311) were sequenced. Sequences of $98 M$. mulatta sampled from 11 provinces of China and Vietnam (GenBank accession nos. AF135271-AF135368, unpublished data by Ding et al.) and $50 \mathrm{M}$. fuscata sampled from six local populations in Japan (GenBank accession nos. AJ419855-AJ419864, Marmi et al., 2004) were included. Localities of samples with known geographic origins are indicated in Fig. 1. 


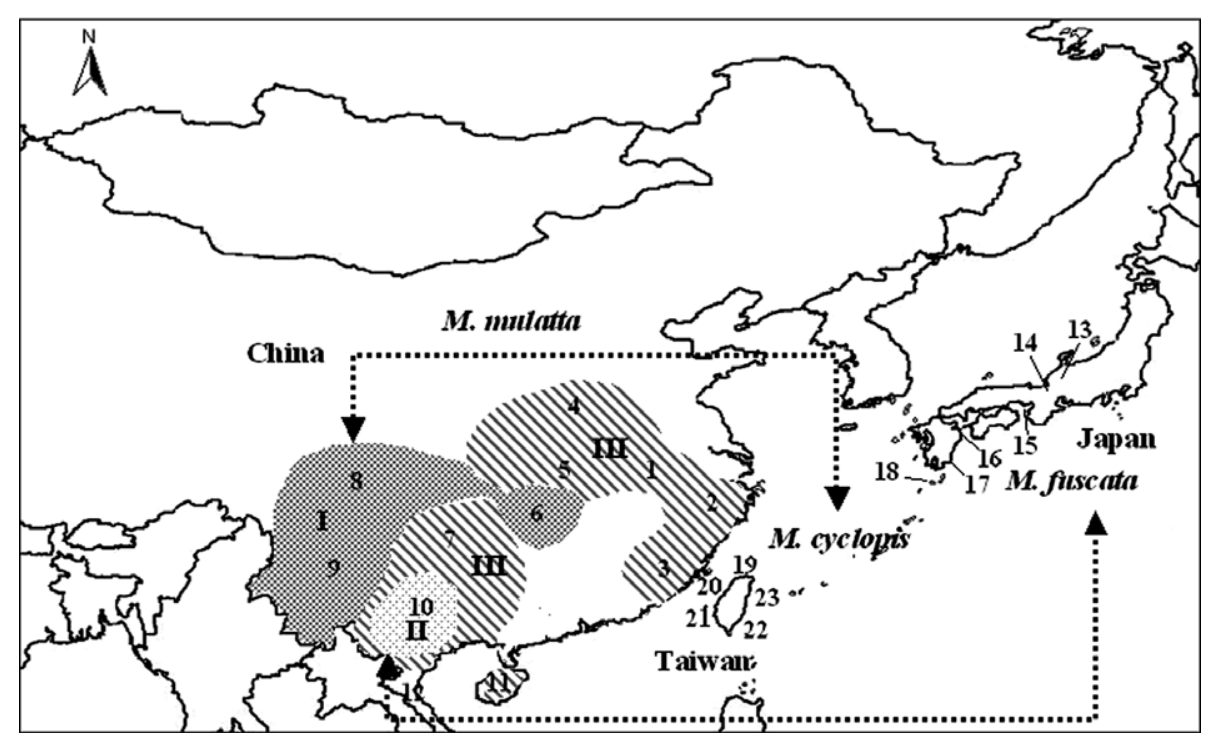

Fig. 1. Sampling localities of Macaca mulatta, M. fuscata and M. cyclopis. (1) Anhui, (2) Zhejiang, (3) Fujian, (4) Henan, (5) Hubei, (6) Hunan, (7) Guizhou, (8) Sichun, (9) Yunnan, (10) Guangxi, (11) Hainan, (12) Vietnam, (13) Hakusan, (14) Takahama, (15) Awajishima, (16) Takasakiyama, (17) Koshima, (18) Yakushima, (19) North Taiwan, (20) Central Taiwan, (21) South Taiwan, (22) East Taiwan and (23) Northeast Taiwan. Geographic distribution of the three phylogenetic clades of $M$. mulatta, haplogroups I, II and III, is also shown on the map. Arrows indicate that $M$. cyclopis and $M$. fuscata may be genetically more related to M. mulatta of haplogroups I and II, respectively.

\subsection{DNA extraction, PCR amplification and nucleotide sequencing}

Total genomic DNA was extracted by traditional phenolchloroform procedure (Kocher et al., 1989). Some of the DNA samples extracted from feces were further eluted by a silica pellet method (Geneclean III, Bio101) when the first PCR attempt failed. The mtDNA CR was amplified by PCR using a primer set of DL1 (forward): 5'-CCAGAAATGAA CACCCTTCCTAGGGC-3' (Chu et al., 2005) and Saru5 (reverse): 5'-GCCA GGACCAAGCCTATTT-3' (Hayasaka et al., 1991) that produced a $1.4 \mathrm{~Kb}$ fragment composed of the whole $\mathrm{CR}$ region and the conservative peripheral regions. The long PCR product may reduce the possibility to amplify a nuclear $\mathrm{CR}$ pseudogene. The PCR mixture contained $10 \mathrm{pmol}$ of each primer, $200 \mu \mathrm{M}$ dNTP, $1.5 \mathrm{mM} \mathrm{MgCl}_{2}, 1 \times$ PCR reaction buffer (20 mM Tris- $\mathrm{HCl}, \mathrm{pH} 8.4,20 \mathrm{mM} \mathrm{KCl})$, 1 unit Taq polymerase (GIBCO BRL) and about $1-3 \mu \mathrm{L}$ DNA extract in a total volume of $50 \mu \mathrm{L}$. A DNA thermal cycler (Biometra) was programmed to perform initial denaturation at $95^{\circ} \mathrm{C}$ for $10 \mathrm{~min}$ and 32 cycles of $1 \mathrm{~min}$ at $95^{\circ} \mathrm{C}$, $1 \mathrm{~min}$ at $58^{\circ} \mathrm{C}$, and $2 \mathrm{~min}$ at $72^{\circ} \mathrm{C}$. A $10 \mathrm{~min}$ final extension at $72^{\circ} \mathrm{C}$ was also included. PCR elutes were partially sequenced by ABI 377 or ABI 3100 automated DNA sequencer using DL1 (forward) and another internal primer DH1 (reverse): 5'-CGGAGATGGGGGTGGGGGGGTTTG-3' (Chu et al., 2005).

\subsection{Analysis of mitochondrial DNA sequences}

Sequences were aligned by Pileup program in GCG (Wisconsin Package Version 10.1, Genetics Computer Group, Madison, WI). A fragment of 386 nucleotide sites was available for analyses after alignment. For a comparison of genetic polymorphism of the four species, nucleotide diversity $(\pi)$ and mean nucleotide difference within species $\left(d_{x}\right)$ were calculated using Arlequin 2.0 (Schneider et al., 2000).

Phylogenetic relationship of unique haplotypes was constructed using neighbor-joining (NJ) method (Saitou and Nei, 1987) and Bayesian approach and was rooted by sequences of $M$. fascicularis. NJ tree was constructed by MEGA 3.1 (Kumar et al., 2004). When constructing phylogenetic trees, distance estimated by a sophisticated model often generates a large variance and thus reduces the resolution of the tree topology when conducting bootstrap (Nei and Kumar, 2000b). The situation could be worse when the dataset is composed of a large number of taxa with short nucleotide sequence (Nei and Kumar, 2000a). To avoid such problem, a simple $p$-distance model was used to calculate genetic distance between distinct sequences. Statistical support of the tree topology was estimated by interior branch analysis with 1000 bootstrap replicates.

Bayesian analysis of the sequence data was performed using MrBayes 3.1 (Huelsenbeck and Ronquist, 2001; Ronquist and Huelsenbeck, 2003), with starting trees generated randomly. A total of 5,000,000 generations were run and the likelihood scores stabilized after 500,000 generations. A tree was saved every 100 generations to give 50,000 trees, of which the first 10,000 were rejected (corresponding to those obtained before the likelihood scores stabilized). Three Markov Chain Monte Carlo runs each having four simultaneous chains were performed independently. The joint posterior probability distribution of each run was congruent, suggesting that the chains were run for a sufficient number of generations to sample the posterior probability landscape adequately. Bootstrap values or likelihood probabilities of $90 \%$ and $70 \%$ were considered as thresholds of high and moderate supports for a clade, respectively. 


\subsection{Demographic inferences}

Mismatch distribution analysis (MDA) was conducted to infer possible population expansion of a species. MDA assumes the pairwise nucleotide differences of haplotypes drawn from a population to have a unimodal distribution if it has experienced a demographic expansion event; otherwise, the distribution is multimodal reflecting a stochastic shape of gene trees (Rogers and Harpending, 1992). Goodness of fit, i.e. the sum of square deviations (SSD) between the observed and the expected mismatch (Schneider and Excoffier, 1999), and Harpending's raggedness index (Harpending, 1994) were calculated to examine the statistical support of the expansion event. Fu's $F_{\mathrm{s}}$ test $(\mathrm{Fu}, 1997)$ was conducted to further confirm the demographic expansion. Because insufficient sampling tends to bias the analysis, each unique haplotype was used once regardless of its frequency of occurrence in samples by assuming the increase of haplotypes refers to a population expansion event (Harpending et al., 1993). The approach may ignore recent expansions but can detect relatively ancient ones.

Time of a confirmed expansion was estimated by $\tau=2 u t_{\mathrm{e}}$, where $\tau$ is estimated by MDA, $u$ is the sum of per nucleotide mutation rate in the DNA region under study and $t_{\mathrm{e}}$ is time since expansion. Mutation rates of the mtDNA CR estimated from various phylogenetic studies (reviewed by Parsons et al., 1997) were in a range of 0.025 0.26 site $^{-1} \mathrm{myr}^{-1}$. In this study, we adopted the medium of the reported values, $0.14 \mathrm{site}^{-1} \mathrm{myr}^{-1}$, which corresponds to a divergence rate of $28 \%$ per million years in the mtDNA CR. Hence, time since expansion was estimated by $t_{\mathrm{e}}$ $(\mathrm{myr})=\tau /\left(2 \times 0.14 \mathrm{site}^{-1} \mathrm{myr}^{-1} \times 386\right.$ sites $)$. A $95 \%$ confidence interval for $\tau$ was obtained by a parametric bootstrap approach (Schneider and Excoffier, 1999).

Initial times of the mtDNA CR divergence between the four species in the fascicularis species group were estimated by $d_{x y}=2 u t_{\mathrm{d}}$, where $d_{x y}$ is the mean nucleotide difference between haplotypes of two species, $t_{\mathrm{d}}$ is time since divergence and $u$ is the same as described above. Because ancestral populations of $M$. cyclopis and M. fuscata may have originated from different local populations of ancient M. mulatta, genetic diversity in each of the two species is supposed to be a subset of genetic diversity of $M$. mulatta in the eastern Asia. Thus, samples of M. mulatta were divided into three groups as revealed from the phylogenetic analysis for further comparison. The initial periods of gene divergence between $M$. cyclopis or M. fuscata and each of the three groups of $M$. mulatta were also estimated by $d_{x y}=2 u t_{\mathrm{d}}$. To correct the discrepancy between "gene divergence" and "population divergence" due to the presence of ancestral polymorphism in populations (Edwards and Beerli, 2000), times of population divergence (i.e., when the two ancestral populations were isolated to each other) between species or populations in the mulatta group were estimated by net nucleotide divergence, $d_{\mathrm{A}}=2 u t$ (Nei and $\mathrm{Li}, 1979)$. The most conservative time of population divergence was estimated from the minimum value of $d_{\mathrm{A}}$. Here
$d_{\mathrm{A}}=d_{x y}-1 / 2\left(d_{x}+d_{y}\right)$, where $d_{x y}$ is the mean nucleotide difference between haplotypes of species (or populations) $x$ and $y, d_{x}$ and $d_{y}$ are the mean nucleotide differences between haplotypes within species (or populations) $x$ and $y$, respectively.

Since the distribution of the haplotypes from eastern China in the phylogenetic tree was not in accordance to their geographic affinity, pairwise nucleotide differences among haplotypes sympatric in Guangxi (14 haplotypes), Zhejiang (eight haplotypes) and Fujian (six haplotypes) provinces were examined for the possible ages of divergence. Distribution of pairwise nucleotide differences was calibrated on a time scale of Pleistocene events (Jenkins, 2001) by $d_{x y}=2 u t_{\mathrm{d}}$.

\section{Results}

\subsection{Mitochondrial DNA CR variation}

Among the total sequences examined $(N=301), 124$ distinct haplotypes were identified. Basic genetic polymorphism parameters of the four species are summarized in Table 1 . In total, 188 variable sites were found, including 169 transitions, 44 transversions, and 20 indels. Nucleotide diversity was the highest in $M$. fascicularis $(0.105 \pm 0.065$, $\pi \pm \mathrm{SD})$, followed by M. mulatta $(0.081 \pm 0.039)$, and was smaller in $M$. fuscata $(0.062 \pm 0.031)$ and in $M$. cyclopis $(0.061 \pm 0.030)$. The trend of genetic diversity agrees with the phylogenetic relationship of the four species that ancestral taxon tends to retain higher genetic diversity than derived taxa.

\subsection{Phylogeny reconstruction}

Branching patterns and the statistic supports in the NJ and the Bayesian trees were similar except for some tip clusters. Hence, only the NJ tree is illustrated in Fig. 2 to show the relationship between haplotypes. The NJ tree comprised five phylogenetic clades with high bootstrap support $(>90 \%)$ and a single lineage (Zhejiang4 of $M$. mulatta) outward the major clades (Fig. 2A). M. cyclopis and M. fuscata each formed a monophyletic clade, while $M$. mulatta comprised three haplogroups (Haplogroup I to III) and the single lineage. The clustering patterns of the haplotypes within the two island species clades corresponded to their geographic affinity (Fig. 2B), while the patterns within the Haplogroups I and

Table 1

Sample size $(N)$, number of haplotypes $(\mathrm{H})$, number of polymorphic sites $(\mathrm{P})$, and nucleotide diversity $(\pi)$ in the $5^{\prime}$ end partial segment of the mtDNA CR of the four Macaca species in the fascicularis species group

\begin{tabular}{lrrrl}
\hline Species & \multicolumn{1}{c}{$\mathrm{N}$} & \multicolumn{1}{c}{$\mathrm{H}$} & \multicolumn{1}{c}{$\mathrm{P}$} & $\pi(\mathrm{SD})$ \\
\hline M. fascicularis & 5 & 5 & 86 & $0.105(0.065)$ \\
M. mulatta & 98 & 57 & 138 & $0.081(0.039)$ \\
M. cyclopis & 148 & 53 & 107 & $0.061(0.030)$ \\
M. fuscata & 50 & 9 & 61 & $0.062(0.031)$ \\
Total & 301 & 124 & 188 & \\
\hline
\end{tabular}


II of M. mulatta corresponded to geographic affinity as well. The Haplogroup I consisted of haplotypes from Hunan, Yunnan and Sichuan in China, while the Haplogroup II comprised of haplotypes from Guangxi in China and Vietnam. As for the Haplogroup III, it contained haplotypes from a wide range from Henan, Hubei, Anhui, Zhejiang, Fujian, Guangxi to Hainan in China and Vietnam (Fig. 1). In this haplogroup, closely related haplotypes were distributed in several provinces of China or Vietnam, and some sympatric haplotypes in Henan, Zhejiang, Fujian, Guangxi, and Vietnam were too divergent to be clustered together (Fig. 2A). In addition, since bootstrap supports among the five phylogenetic clades were less than $90 \%$, the relationship of these clades could not be concluded. However, under the

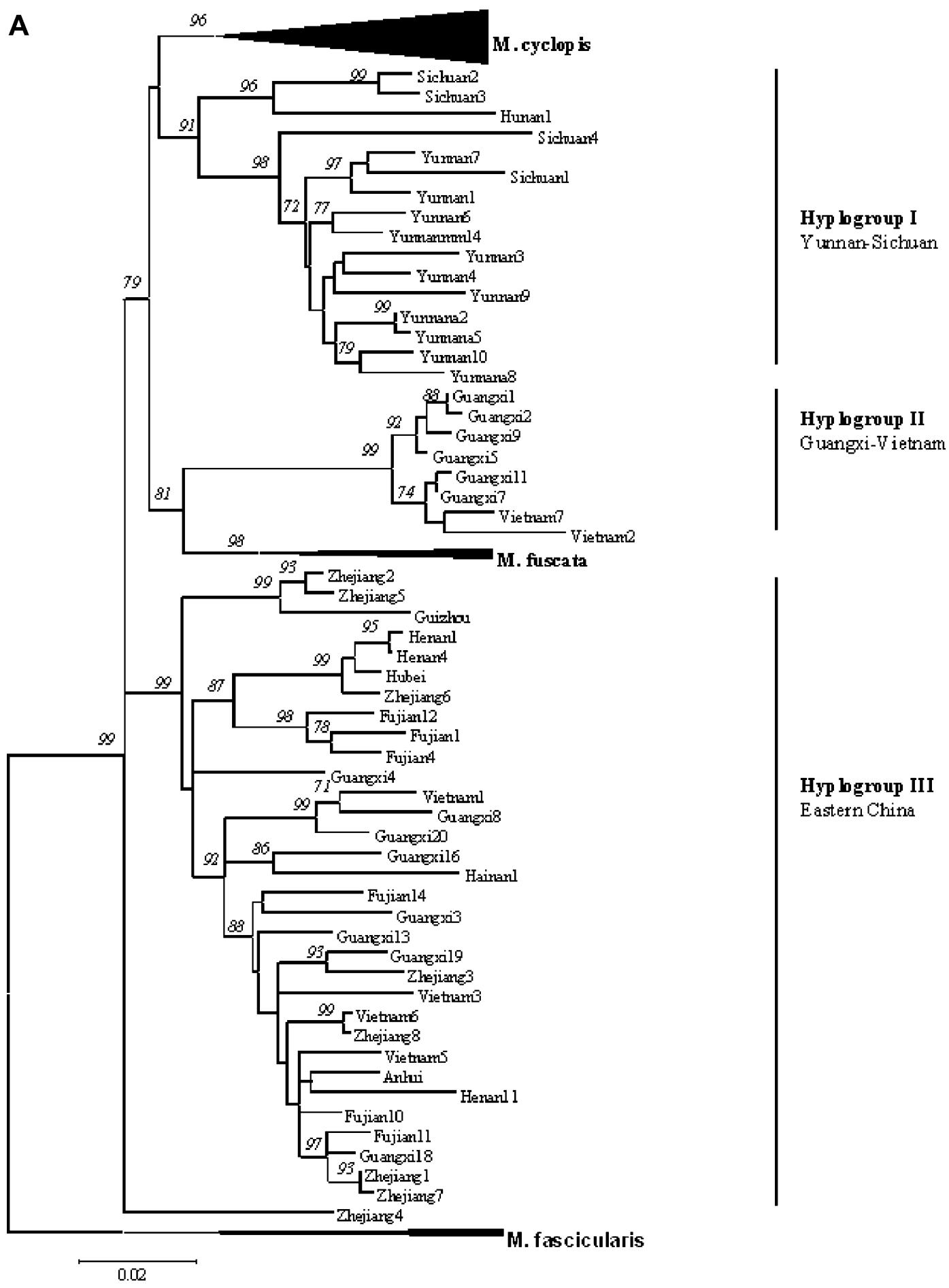

Fig. 2. Neighbor-joining tree illustrating the phylogenetic relationship among the $124 \mathrm{mtDNA}$ CR haplotypes of the four Macaca species in the fascicularis species group. The percentage bootstrap support (1000 replicates) greater than 70 is listed for interior branches. The haplogroups of $M$. cyclopis (53 haplotypes) and $M$. fuscata (nine haplotypes) are compressed in (A) and the haplogroups of $M$. mulatta are compressed in (B) in order to simplify the tree topology. M. fascicularis (five haplotypes) is the outgroup to root the tree. Haplotype codes of M. mulatta follow Ding et al. published in the Genbank. 


\section{B \\ B}
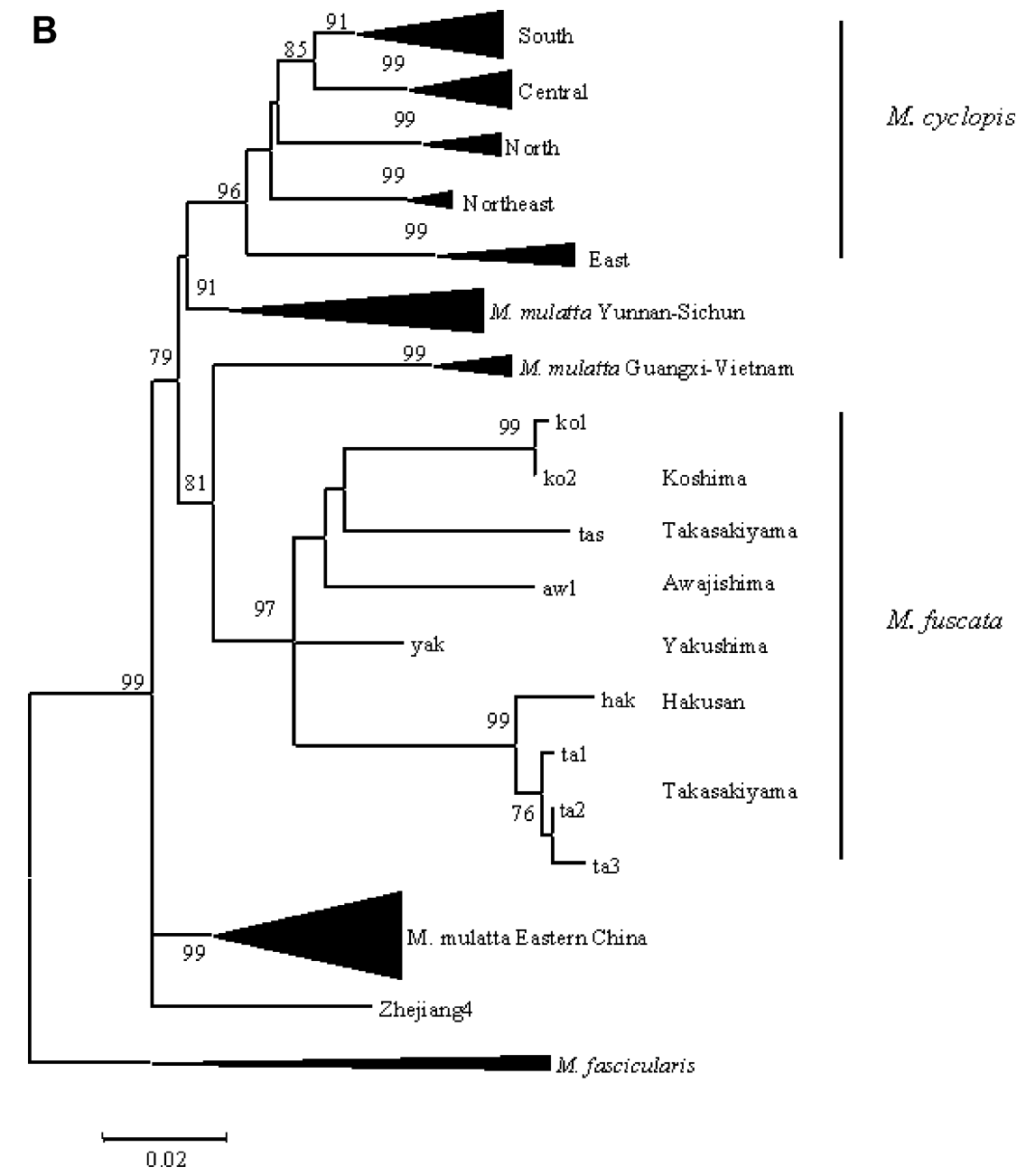

M. cyclopis

Fig. 2 (continued)

criterion of moderate support (bootstrap value $>70 \%$ ), $M$. fuscata was grouped with Haplogroup II into a cluster (bootstrap value $=81 \%$ ), which belonged to a larger assemblage with M. cyclopis and the Haplogroup I included (bootstrap value $=79 \%$ ).

\subsection{Demographic inferences}

Statistical parameters of MDA did not reject a smooth, unimodal distribution of pairwise nucleotide differences between unique haplotypes in each of the four species in the fascicularis species group (Table 2). It indicated that population expansion occurred in all of the four species. Only the ages of expansion in M. mulatta and M. cyclopis were described because samples of $M$. fascicularis (five haplotypes) and $M$. fuscata (nine haplotypes) were under-represented. Mismatch distributions of $M$. mulatta and $M$. cyclopis are illustrated in Fig. 3. Expansion events of the two species were further confirmed by the large negative values of Fu's $F_{\mathrm{s}}$ (Table 2). The values of $\tau$ were 33.5 in M. mulatta and 23.9 in $M$. cyclopis, which gave the estimated ages of expansion to be 0.31 mya (95\% CI of $\left.t_{\mathrm{e}}=0.24-0.41 \mathrm{mya}\right)$ and 0.22 mya (95\% CI of $t_{\mathrm{e}}=0.15-0.37$ mya) for the two species, respectively.

The mean number of nucleotide differences $\left(d_{x}\right)$ within species showed the same decreasing trend from the basal taxon

Table 2

Population expansion parameters and Fu's $F_{\mathrm{s}}$ test of the four Macaca species in the fascicularis species group

\begin{tabular}{llccrr}
\hline Species & $\tau(95 \% \mathrm{CI})$ & $t^{\mathrm{a}}(95 \% \mathrm{CI})$ & $\mathrm{SSD}^{\mathrm{b}}(p)$ & $\mathrm{RI}^{\mathrm{c}}(p)$ & $\mathrm{Fu}^{\prime} F_{\mathrm{s}}(p)$ \\
\hline M. fascicularis & $49.5(35.4-62.1)$ & $0.46(0.33-0.57)$ & $0.075(p=0.75)$ & $0.200(p=0.53)$ & $1.27(p=0.47)$ \\
M. mulatta & $33.5(26.2-44.8)$ & $0.31(0.24-0.41)$ & $0.002(p=0.83)$ & $0.001(p=1.00)$ & $-24.13(p<0.01)$ \\
M. cyclopis & $23.9(16.6-40.2)$ & $0.22(0.15-0.37)$ & $0.004(p=0.34)$ & $0.003(p=0.70)$ & $-24.12(p<0.01)$ \\
M. fuscata & $30.6(23.1-36.0)$ & $0.28(0.21-0.33)$ & $0.035(p=0.25)$ & $0.099(p=0.14)$ & $-1.08(p=0.18)$ \\
\hline
\end{tabular}

a The time unit of $t$ is in myr.

b SSD: The sum of square deviations between the observed and the expected mismatch (Schneider and Excoffier, 1999).

c RI: Harpending's raggedness index (Harpending, 1994). 
A M. mulatia

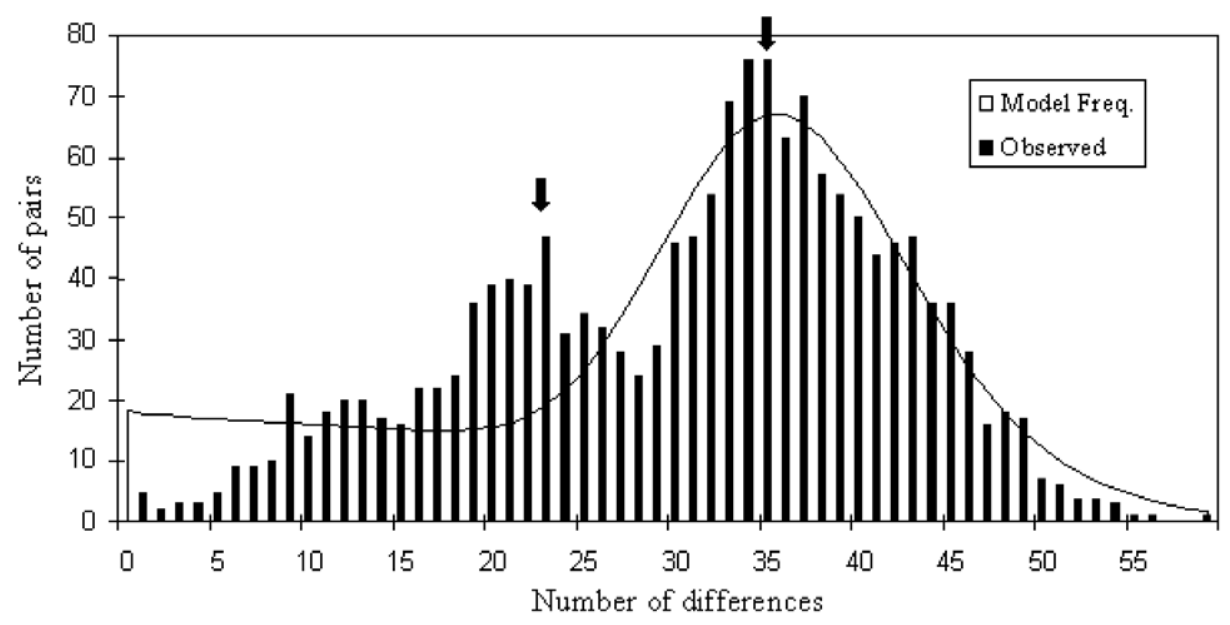

B M. cyclopis

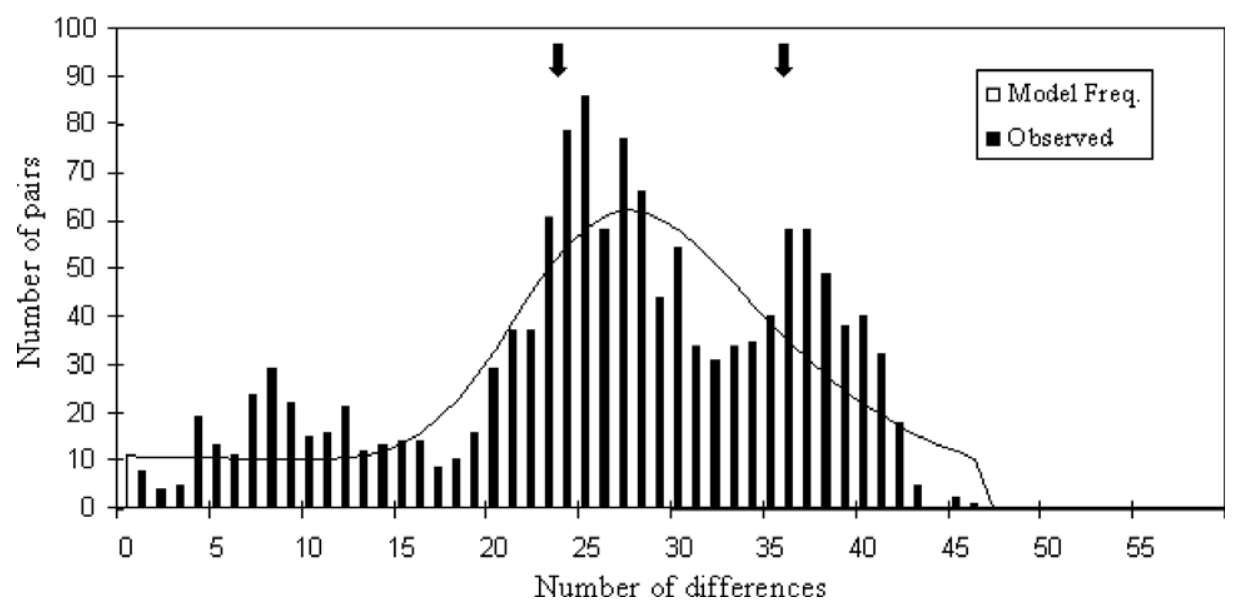

Fig. 3. Observed and expected mismatch (model frequency) distributions given the null distribution (unimodal, bell-shaped) corresponding to population expansion for (A) M. mulatta, and (B) M. cyclopis. Arrows indicate similar modes of population expansion.

(M. fascicularis, $d_{x}=40.5$ ) to the tip taxa (M. cyclopis and $M$. fuscata, $d_{x}=26.2$ and 24.7, respectively) in this species group (Table 3). The values of $d_{x y}$ between M. cyclopis and each of the three haplogroups of M. mulatta ranged from 42.7 to 47.1 (Table 3), which corresponded to an initial gene divergent time of $0.40-0.44$ mya. Similarly, the values of $d_{x y}$ between $M$. fuscata and each of the three haplogroups of M. mulatta ranged from 40.8 to 45.4 , which corresponded to an initial gene divergent time of $0.38-0.42$ mya. The minimum values of $d_{\mathrm{A}}$ between M. cyclopis or M. fuscata and M. mulatta were 18 and 19.5 (Table 4), which indicated the most conservative time of population divergence to be 0.17 and 0.18 mya, respectively. In addition, various divergent levels were found between hap- lotypes sympatric in Guangxi, Zhejiang and Fujian. The pairwise nucleotide differences of these sympatric haplotypes showed coincident modes around 0.01, 0.06-0.12 and 0.180.32 mya (Fig. 4), the timing of which approximated to various interglacial periods in the Pleistocene.

\section{Discussion}

Previous authors have reported mtDNA paraphyly among mulatta lineages from India and China (Hayasaka et al., 1996; Melnick et al., 1993; Morales and Melnick, 1998; Tosi et al., 2003). We also found that M. mulatta is not a mitochondrially monophyletic taxon. However,

Table 3

Mean number of nucleotide differences among $\left(d_{x y}\right.$, above diagonal), within ( $d_{x}$, diagonal) and net nucleotide differences among $\left(d_{\mathrm{A}}\right.$, below diagonal) the four Macaca species in the fascicularis group in a segment of 386 nucleotide sites of the mtDNA CR

\begin{tabular}{lllll}
\hline & M. fascicularis & M. mulatta & M. cyclopis & M. fuscata \\
\hline M. fascicularis & 40.5 & 59.8 & 62.4 & 66.2 \\
M. mulatta & 24.2 & 30.8 & 43.2 & 44.1 \\
M. cyclopis & 29.0 & 14.7 & 26.2 & 45.6 \\
M. fuscata & 33.6 & 16.3 & 20.4 & 24.7 \\
\hline
\end{tabular}


Table 4

Mean number of nucleotide differences among $\left(d_{x y}\right.$, above diagonal), within $\left(d_{x}\right.$, diagonal) and net nucleotide differences among $\left(d_{\mathrm{A}}\right.$, below diagonal) the five Macaca populations in the mulatta group in a segment of 386 nucleotide sites of the mtDNA CR

\begin{tabular}{|c|c|c|c|c|c|}
\hline & M. cyclopis & M. mulatta I & M. mulatta II & M. mulatta III & M. fuscata \\
\hline M. cyclopis & 26.2 & 42.7 & 47.1 & 42.8 & 45.8 \\
\hline M. mulatta $\mathrm{I}^{\mathrm{a}}$ & 18.0 & 23.2 & 44.7 & 38.5 & 43.4 \\
\hline M. mulatta $\mathrm{II}^{\mathrm{b}}$ & $\overline{30.2}$ & 29.2 & 7.7 & 34.7 & 40.8 \\
\hline M. fuscata & 20.4 & 19.5 & 24.6 & 22.7 & 24.7 \\
\hline
\end{tabular}

Minimum values of $d_{\mathrm{A}}$ between $M$. cyclopis or M. fuscata to each of the three haplogroups of $M$. mulatta are underlined.

${ }^{\text {a }}$ Haplogroup I (Yunnan-Sichun).

${ }^{\mathrm{b}}$ Haplogroup II (Guangxi-Vietnam).

${ }^{c}$ Haplogroup III (Eastern China).

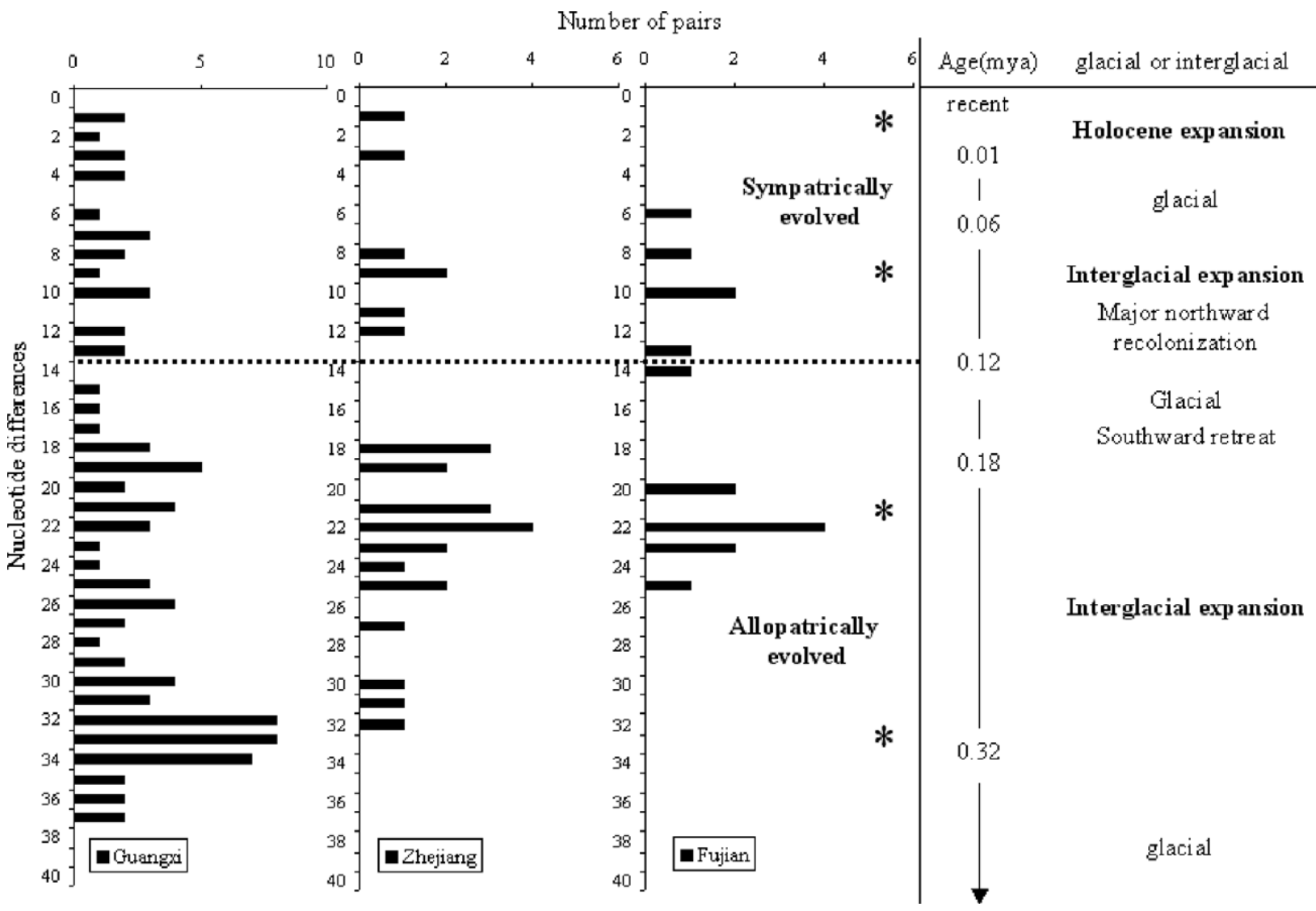

Fig. 4. Distribution of pairwise nucleotide differences of $M$. mulatta haplotypes in Guangxi, Zhejiang, and Fujian provinces of China aligned with the time frame of glacial and interglacial periods in the Pleistocene. Dotted line indicates the pairwise nucleotide difference of 13, corresponding to divergence around 0.12 mya. Sympatric haplotypes differing by more than 13 nucleotides may have evolved allopatrically. Asterisks indicate possible expansion modes during interglacial periods in the Pleistocene.

although we did not have the sequences from India-Pakistan to re-examine the reported pattern, our result revealed a different paraphyletic pattern in mtDNA among mulatta lineages unreported elsewhere. By applying a highly polymorphic mtDNA marker and a larger panel of mtDNA sequences than previous studies (Deinard and Smith, 2001; Hayasaka et al., 1996; Morales and Melnick, 1998; Tosi et al., 2000; 2003; Zhang and Shi, 1993), we found that western (Yunnan-Sichuan) and eastern Chinese lineages were separated from each other in the phylogenetic tree with respect to M. cyclopis and M. fuscata (Fig. 2A). The result supports the recent divergence of $M$. cyclopis and $M$. fuscata from Chinese M. mulatta and the retention of genetic polymorphism in the Chinese $M$. mulatta as described elsewhere (Hoelzer, 1997; Melnick et al., 1993; Morales and Melnick, 1998). Moreover, the monophyly of $M$. cyclopis and $M$. fuscata suggests postglacial vicariance after the ancestral mulatta populations dispersed into Taiwan and Japan, respectively.

\subsection{Retreat-recolonization history of M. mulatta}

In this study, no distinct geographic pattern was found in haplotypes of $M$. mulatta distributed in the eastern 
China (Fig. 2A). Multiple divergent mtDNA lineages (especially those differing by more than 20 nucleotides) were found sympatric in Henan, Zhejiang, Fujian and Guangxi provinces of China and Vietnam, but no haplotype closely related to these lineages in nearby localities. It shows a discrepant phylogeographic pattern against isolation by distance (IBD) model (Wright, 1943). By assuming mtDNA haplotypes accumulate during population expansion and a mutation rate of 0.14 site $^{-1} \mathrm{myr}^{-1}$ in the mtDNA CR, possible nucleotide differences between sympatric haplotypes evolved from a single mtDNA matriline during different temporal ranges can be estimated. Applying the time frame of glacial and interglacial events described by Jenkins (2001), the divergent time of haplotypes differing by about two nucleotides is less than 0.01 my (i.e. during Holocene). Accordingly, differences of 6-13 and more than 20 nucleotides between haplotypes correspond to divergence since the last and the second last interglacials $(0.06-0.12$ mya and $>0.2$ mya), respectively (Fig. 4).

However, it is less likely for multiple mtDNA lineages to coexist for such a long time under the process of lineage sorting (Avise et al., 1984; Hoelzer et al., 1998; Wallman et al., 1996) when effective population size decreased dramatically during glacial periods (e.g. 0.01-0.06 mya and 0.12-0.18 mya, etc.). Moreover, even if these divergent lineages evolved sympatrically from the same mtDNA matriline, phylogenetic relationship between them should still follow the IBD model not observed in this study. Hence, it is less likely that these divergent mtDNA haplotypes evolved sympatrically.

Two other scenarios may lead to sympatry of multiple divergent mtDNA lineages observed in this study. It may have resulted either from southward retreat that caused a stochastic collection of divergent lineages in a locality (i.e. a refugium) during glacial period, or from population expansion that brought divergent mtDNA lineages into contact during interglacial period. In the former case, it infers "forced dispersal" for populations characterized by female philopatry. A great scale of mtDNA lineage extinction and range shift may have occurred during the retreat process due to competition or simply deteriorated environments. A corollary to it is a great gap of nucleotide difference between sympatric haplotypes and the lack of closely related haplotypes nearby. In the latter case, it infers secondary admixture due to population expansion. Since population expansion tends to maintain mtDNA lineages, haplotypes closely related to these divergent mtDNA lineages can be found in nearby populations.

Results in this study indicate that phylogenetic relationship of haplotypes distributed in the eastern China is against the IBD model. Multiple divergent mtDNA lineages were found coexisting in several localities without closely related lineages nearby. This suggests a complex amalgamation of distant mtDNA lineages due to the southward retreat in the eastern China. Thus, the genetic data supports that there have been a southward retreat as proposed by Fooden and Albrecht (1999). In addition, it also indicates that possible glacial refugia may include regions in Zhejiang, Fujian and Guangxi provinces that harbor divergent mtDNA lineages in China (probably in the mountain regions such as Wuyi-Shan and Nan Ling).

Fooden and Albrecht (1999) proposed that the southward retreat and the subsequent recolonization of $\mathrm{M}$. $\mathrm{mul}$ atta in the eastern Asia occurred during and after the LGM (0.018 mya), respectively. If ages of the two events were as proposed, the dispersal pattern of retreat may be hard to trace, while that of recolonization should be quite apparent. The resulting pattern should be some closely related mtDNA haplotypes (differed by one or two nucleotides) overlaying in a wide range (e.g. Chen et al., 2005; Conroy and Cook, 2000; Fu et al., 2005; Hatase et al., 2002; Ritchie et al., 2004; Ruedi and Castella, 2003). However, we did not detect any such pattern. Instead, geographic affinity observed in most sub-clades consisting of haplotypes differing by less than 13 nucleotides suggests to date the recolonization event back to a more ancient age. In addition, nucleotide difference between those sympatric but divergent mtDNA lineages ( $>20$ nucleotides) also suggests a more ancient time of the retreat event than during LGM. Hence, the scenario presented by Fooden and Albrecht (1999) was supported by our genetic data, however, the chronology of the scenario is arguable. In the following, we propose plausible ages for the southward retreat and the northward recolonization of $M$. mulatta, based on the divergent level of mtDNA CR haplotypes.

First, the major southward retreat, which generated a complex pattern of CR haplotypes in the eastern China, occurred during the second last glacial $(0.12-0.18$ mya or earlier, corresponding to nucleotide difference $>13$ between sympatric haplotypes) instead of the LGM (0.018 mya). The retreat apparently displaced the native populations (the long-tailed mulatta populations) inhabiting the $15^{\circ} \mathrm{N}-$ $25^{\circ} \mathrm{N}$ latitudinal zone and resulted in a discrepant phylogenetic relationship between $M$. cyclopis and $M$. mulatta. That is, $M$. cyclopis is not mitochondrially more related to M. mulatta in the eastern China (such as populations in Fujian and in Zhejiang). Instead, it is more related to $M$. mulatta in the southwest China (Yunnan, Haplogroup I) (Figs. 1 and 2). However, the connection between M. cyclopis and the Haplogroup $\mathrm{I}$ is not well supported (bootstrap $=28 \%$ ) probably due to the extinction of the "transitional populations". The extant populations of $M$. mulatta in Yunnan and M. cyclopis may be two relictual groups descended from ancestors more similar to the longtailed mulatta populations. The mountain area in the southwestern China proposed as a refugium for various animals and plants in China (Harrison et al., 2002) has protected Yunnan populations from displacement. Nonetheless, a decreasing west-east gradient of RTL from $90^{\circ} \mathrm{E}$ to $115^{\circ} \mathrm{E}$ (Fooden, 2000) and a more or less homogeneous pattern of allozymes (Fooden and Lanyon, 1989) and microsatellites (Morin et al., 1997) in M. mulatta indicate that male-mediated gene flow from the eastern China population to the Yunnan population was common. Besides, postglacial 
vicariance may prevent gene flow between China and Taiwan and thus retained the morphological character of a longer tail in M. cyclopis.

Subsequently, during the last interglacial (0.06-0.12 mya, corresponding to nucleotide differences about 6-13 between haplotypes), the major northward recolonization occurred that covered the current range of M. mulatta in China. Population expansion during this period can be inferred from a similar level of lineage divergence within provinces of Yunnan and Fujian, as well as between Guangxi, Fujian, Zhejiang and Vietnam. During the last glacial, populations may have been isolated in multiple refugia located in Henan, Zhejiang, Fujiang and Guangxi, instead of performing another large-scaled retreat. Similar locations of glacial refugia in the southern China have also been suggested for a conifer species, Cunninghamia konishii ( $\mathrm{Lu}$ et al., 2001).

Afterward, the Holocene expansion was mostly confined locally. It was inferred from the observation that the same or related haplotypes were found within provinces of China, e.g. haplotypes of Haplogroup II in Guangxi, haplotypes in Zhejiang, Henan, and Yunnan (Fig. 2A). However, the occurrence of the same or related haplotypes in distant localities provides some exceptions (detailed data not showed). For example, haplotype Sichuan1 occurs in Sichun and Hainen, while haplotype Yunnana5 occurs in Yunnan and Hunan, as well as two closely related haplotypes, Zhejiang9 and Vietnam6, occur in two distant localities Zhejiang and Vietam. The exceptions need to be reexamined for the possibility of anthropogenic introduction.

\subsection{Divergence and demographic history of ancient mulatta populations}

Mean nucleotide divergences between $M$. mulatta and each of the two island species, M. cyclopis and M. fuscata $\left(d_{x y}\right.$, Table 3$)$, suggest that ancestral mulatta populations colonized Taiwan and Japan in approximately the same time period around $0.38-0.44$ mya during the early stage of the third last glacial (0.32-0.44 mya). The result is also supported by fossil records that suggest the existence of $M$. cyclopis in Taiwan and M. fuscata in Japan at least 0.3 mya (Fooden and $\mathrm{Wu}, 2001$ ) and 0.4 mya (Kamei, 1969), respectively. Considering ancestral polymorphism, the most conservative estimates of the population divergent time between $M$. mulatta and each of the two species are around 0.17 and 0.18 mya, respectively. The estimates indicate the times of population (or species) isolation were during the end of the second last interglacial (0.18-0.32 mya) and the beginning of the second last glacial (0.12-0.18 mya). The observed monophyly in M. cyclopis and M. fuscata suggests that ancestral population of each species originated from different regional population in mainland at around 0.4 mya, and, during 0.17-0.4 mya, dispersed from mainland to Taiwan or Japan when dispersal routes were available. Furthermore, the divergent level between the three species also indicates that successful dispersal was unlikely since the second last glacial. It is concordant with the palynological evidence that most of the exposed continental shelf connecting Taiwan and China was covered by savanna-like vegetation (Liew et al., 1998) that is not suitable for macaques. The cool and arid climate even extended to a lower latitudinal zone of the Sunda region (GathorneHardy et al., 2002; Heaney, 1991).

The MDA did not reject a smooth, unimodal distribution of nucleotide differences between haplotypes in $M$. mulatta and M. cyclopis. It suggests that the population expansion occurred around 0.22 and 0.31 mya for M. cyclopis and M. mulatta, respectively (Table 2). Besides, there are multiple modes (arrows placed in Fig. 3) embedded in the empirical curves of both species. The similarity of the modal patterns in the two species indicates that they have experienced similar demographic history during Pleistocene. The expansion that dated back to a more ancient past (0.31 mya) also indicates earlier population colonization.

Molecular dating of any event (e.g. divergence of populations or population expansion) is subject to the referred mutation rate and needs to be interpreted with caution (Hillis et al., 1996). Nevertheless, the estimated ages of population expansion in the study are consistent with the glacial history, and the divergent times between $M$. cyclopis, M. fuscata and M. mulatta are within the ranges reported elsewhere (Hayasaka et al., 1996; Marmi et al., 2004; Morales and Melnick, 1998; Tosi et al., 2003). The hypothetic scenario suggested by Fooden and Albrecht (1999) is supported by our mtDNA CR data. However, the ages of the retreat and the recolonization of ancestral $M$. mulatta may be dated back to a more ancient time during the late middle Pleistocene (0.13-0.18 mya) and late Pleistocene (0.06-0.12 mya) instead of the LGM (0.018 mya). In addition, by applying the net nucleotide divergence $\left(d_{\mathrm{A}}\right.$, Nei and $\mathrm{Li}, 1979)$ and a more complete panel of mtDNA sequences than previous studies, a more recent population divergent time, 0.17 mya, for the three species of the mulatta group is suggested.

\section{Acknowledgments}

We would like to acknowledge S.W. Chang, K.F. Lin, S.M. Chen, M.W. Fan, Wildlife labs of NDHU and NTU for their assistance of sample collection. GCG program service is kindly provided by National Healthy Research Institutes (NHRI) in Taiwan. The research was supported by National Science Council, Taiwan (NSC-89-2311-B-002082) and the fund of the Institute of Zoology, Academia Sinica to H.Y. Wu.

\section{References}

Avise, J.C., Neigel, J.E., Arnold, J., 1984. Demographic influences on mitochondrial DNA lineage survivorship in animal populations. J. Mol. Evol. 20, 99-105.

Avise, J.C., Arnold, J., Ball, R.M., Bermingham, E., Lamb, T., Neigel, J.E., Reeb, C.A., Saunders, N.C., 1987. Intraspecific phylogeography: the 
mitochondrial DNA bridge between population genetics and systematics. Annu. Rev. Ecol. Syst. 18, 489-522.

Brandon-Jones, D., Eudey, A.A., Geissmann, T., Groves, C.P., Melnick, D.J., Morales, J.C., Shekelle, M., Stewart, C.B., 2004. Asian primate classification. Int. J. Primatol. 25, 97-164.

Chen, S.Y., Su, Y.H., Wu, S.F., Sha, T., Zhang, Y.P., 2005. Mitochondrial diversity and phylogeographic structure of Chinese domestic goats. Mol. Phylogenet. Evol., 37, 804-814.

Chu, J.H., Wu, H.Y., Lin, Y.S., 2005. Mitochondrial DNA diversity in two populations of Taiwanese macaque (Macaca cyclopis). Conserv. Genet. 6, 101-109.

Conroy, C.J., Cook, J.A., 2000. Phylogeography of a post-glacialcolonizer: Microtus longicaudus (Rodentia: Muridae). Mol. Ecol. 9, 165-175.

Deinard, A., Smith, D.G., 2001. Phylogenetic relationships among the macaques: evidence from the nuclear locus NRAMP1. J. Hum. Evol. 41, 45-59.

Delson, E., 1980. Fossil macaques, phyletic relationships and a scenario of deployment. In: Lindburg, D.G. (Ed.), The Macaques: Studies in Ecology, Behavior, and Evolution. Van Nostrand Reinhold, New York, pp. $10-30$.

Edwards, S.V., Beerli, P., 2000. Perspective: gene divergence, population divergence, and the variance in coalescence time in phylogeographic studies. Evolution 54, 1839-1854.

Fooden, J., 1976. Provisional classification and key to living species of macaques (Primates Macaca). Folia Primatol. 25, 225-236.

Fooden, J., 1997. Tail length variation in Macaca fascicularis and $\mathrm{M} \mathrm{mul-}$ atta. Primates 38, 221-231.

Fooden, J., 2000. Systematic Review of the Rhesus Macaque, Macaca mulatta (Zimmermann, 1780). Field Museum of Natural History, Chicago.

Fooden, J., Albrecht, G.H., 1999. Tail-length evolution in fascicularis-group macaques (Cercopithecidae: Macaca). Int. J. Primatol. 20, 431-440.

Fooden, J., Lanyon, S.M., 1989. Blood-protein allele frequencies and phylogenetic-relationships in Macaca - a review. Am. J. Primatol. 17, 209-241.

Fooden, J., Wu, H.-Y., 2001. Systematic review of the Taiwanese macaque, Macaca cyclopis Swinhoe, 1863. Field Museum of Natural History, Chicago.

Fu, Y.X., 1997. Statistical tests of neutrality of mutations against population growth, hitchhiking and background selection. Genetics 147, 915 925.

Fu, J., Weadick, C.J., Zeng, X., Wang, Y., Liu, Z., Zheng, Y., Li, C., Hu, Y., 2005. Phylogeographic analysis of the Bufo gargarizans species complex: A revisit. Mol. Phylogenet. Evol. 37, 202-213.

Gathorne-Hardy, F.J., Syaukani, Davies, R.G., Eggleton, P., Jones, D.T., 2002. Quaternary rainforest refugia in south-east Asia: using termites (Isoptera) as indicators. Biol. J. Linn. Soc. Lond. 75, 453-466.

Groves, C.P., 2001. Primate Taxonomy. Smithsonian Institution Press, Washington, DC.

Hayasaka, K., Fujii, K., Horai, S., 1996. Molecular phylogeny of macaques: Implications of nucleotide sequences from an 896-base pair region of mitochondrial DNA. Mol. Biol. Evol. 13, 1044-1053.

Harpending, H.C., 1994. Signature of ancient population-growth in a lowresolution mitochondrial-DNA mismatch distribution. Hum. Biol. 66, 591-600.

Harpending, H.C., Sherry, S.T., Rogers, A.R., Stoneking, M., 1993. The genetic-structure of ancient human-populations. Curr. Anthropol. 34, 483-496.

Harrison, T., Ji, X.P., Su, D., 2002. On the systematic status of the late Neogene hominoids from Yunnan Province, China. J. Hum. Evol. 43, 207-227.

Hatase, H., Kinoshita, M., Bando, T., Kamezaki, N., Sato, K., Matsuzawa, Y., Goto, K., Omuta, K., Nakashima, Y., Takeshita, H., Sakamoto, W., 2002. Population structure of loggerhead turtles, Caretta caretta, nesting in Japan: bottlenecks on the Pacific population. Mar. Biol. 141, 299-305.

Hayasaka, K., Ishida, T., Horai, S., 1991. Heteroplasmy and polymorphism in the major noncoding region of mitochondrial-DNA in Japanese monkeys-association with tandemly repeated sequences. Mol. Biol. Evol. 8, 399-415.

Heaney, L.R., 1991. A synopsis of climatic and vegetational change in Southeast-Asia. Clim. Change 19, 53-61.
Hillis, D.M., Moritz, C., Mable, B.K., 1996. Molecular Systematics. Sinauer Associates, Sunderland, MA

Hoelzer, G.A., 1997. Inferring phylogenies from mtDNA variation: Mitochondrial-gene trees versus nuclear-gene trees revisited. Evolution 51, 622-626.

Hoelzer, G.A., Wallman, J., Melnick, D.J., 1998. The effects of social structure, geographical structure, and population size on the evolution of mitochondrial DNA: II. Molecular clocks and the lineage sorting period. J. Mol. Evol. 47, 21-31.

Huelsenbeck, J.P., Ronquist, F., 2001. MRBAYES: Bayesian inference of phylogenetic trees. Bioinformatics 17, 754-755.

Jenkins, I., 2001. The Pleistocene. In: Atlas of the Evolving Earth. Macmillan Reference USA, New York, pp. 70-93.

Kamei, T., 1969. Mammals of the glacial age in Japan-especially on Japanese monkey. Monkey 106, 5-12.

Kocher, T.D., Thomas, W.K., Meyer, A., Edwards, S.V., Paabo, S., Villablanca, F.X., Wilson, A.C., 1989. Dynamics of mitochondrial-DNA evolution in animals - amplification and sequencing with conserved primers. Proc. Natl. Acad. Sci. USA 86, 6196-6200.

Kumar, S., Tamura, K., Nei, M., 2004. MEGA3: Integrated software for molecular evolutionary genetics analysis and sequence alignment. Brief Bioinformat. 5, 150-163.

Li, Q.Q., Zhang, Y.P., 2005. Phylogenetic relationships of the macaques (Cercopithecidae: Macaca), inferred from mitochondrial DNA sequences. Biochem. Genet. 43, 375-386.

Liew, P.M., Kuo, C.M., Huang, S.Y., Tseng, M.H., 1998. Vegetation change and terrestrial carbon storage in eastern Asia during the Last Glacial Maximum as indicated by a new pollen record from central Taiwan. Glob. Planet. Change 17, 85-94.

Lu, S.Y., Peng, C.I., Cheng, Y.P., Hong, K.H., Chiang, T.Y., 2001. Chloroplast DNA phylogeography of Cunninghamia konishii (Cupressaceae), an endemic conifer of Taiwan. Genome 44, 797-807.

Marmi, J., Bertranpetit, J., Terradas, J., Takenaka, O., Domingo-Roura, X., 2004. Radiation and phylogeography in the Japanese macaque, Macaca fuscata. Mol. Phylogenet. Evol. 30, 676-685.

Melnick, D.J., Hoelzer, G.A., Absher, R., Ashley, M.V., 1993. MtDNA diversity in rhesus monkeys reveals overestimates of divergence time and paraphyly with neighboring species. Mol. Biol. Evol. 10, 282-295.

Morales, J.C., Melnick, D.J., 1998. Phylogenetic relationships of the macaques (Cercopithecidae: Macaca), as revealed by high resolution restriction site mapping of mitochondrial ribosomal genes. J. Hum. Evol. 34, 1-23.

Morin, P.A., Kanthaswamy, S., Smith, D.G., 1997. Simple sequence repeat (SSR) polymorphisms for colony management and population genetics in rhesus macaques (Macaca mulatta). Am. J. Primatol. 42, 199-213.

Nei, M., Kumar, S., 2000a. Accuracies and statistical tests of phylogenetic trees. In: Molecular Evolution and Phylogenetics. Oxford University Press, New York, pp. 165-186.

Nei, M., Kumar, S., 2000b. Evolutionary change of DNA sequences. In: Molecular Evolution and Phylogenetics. Oxford University Press, New York, pp. 33-50.

Nei, M., Li, W.H., 1979. Mathematical model for studying genetic variation in terms of restriction endonucleases. Proc. Natl. Acad. Sci. USA 76, 5269-5273.

Parsons, T.J., Muniec, D.S., Sullivan, K., Woodyatt, N., AllistonGreiner, R., Wilson, M.R., Berry, D.L., Holland, K.A., Weedn, V.W., Gill, P., Holland, M.M., 1997. A high observed substitution rate in the human mitochondrial DNA control region. Nat. Genet. 15, 363-368.

Ritchie, P.A., Millar, C.D., Gibb, G.C., Baroni, C., Lambert, D.M., 2004 Ancient DNA enables timing of the Pleistocene origin and Holocene expansion of two Adelie penguin lineages in Antarctica. Mol. Biol. Evol. 21, 240-248.

Rogers, A.R., Harpending, H., 1992. Population-growth makes waves in the distribution of pairwise genetic-differences. Mol. Biol. Evol. 9, 552-569.

Ronquist, F., Huelsenbeck, J.P., 2003. MrBayes 3: Bayesian phylogenetic inference under mixed models. Bioinformatics 19, 1572-1574.

Ruedi, M., Castella, V., 2003. Genetic consequences of the ice ages on nurseries of the bat Myotis myotis: a mitochondrial and nuclear survey. Mol. Ecol. 12, 1527-1540. 
Saccone, C., Lanave, C., Pesole, G., Sbisa, E., 1993. Peculiar features and evolution of mitochondrial genome in mammals. In: DiMauro, S., Wallace, D. (Eds.), Mitochondrial DNA in Human Pathology. Raven Press, New York, pp. 27-37.

Saitou, N., Nei, M., 1987. The neighbor-joining method-a new method for reconstructing phylogenetic trees. Mol. Biol. Evol. 4, 406-425.

Schneider, S., Excoffier, L., 1999. Estimation of past demographic parameters from the distribution of pairwise differences when the mutation rates very among sites: Application to human mitochondrial DNA. Genetics 152, 1079-1089.

Schneider, S., Roessli, D., Excoffier, L., 2000. ARLEQUIN: A software for population genetics data analysis, version 2.0. Genetics and Biometry Laboratory, University of Geneva.

Tosi, A.J., Morales, J.C., Melnick, D.J., 2000. Comparison of Y chromosome and mtDNA phylogenies leads to unique inferences of macaque evolutionary history. Mol. Phylogenet. Evol. 17, 133-144.
Tosi, A.J., Morales, J.C., Melnick, D.J., 2002. Y-chromosome and mitochondrial markers in Macaca fascicularis indicate introgression with Indochinese M. mulatta and a biogeographic barrier in the Isthmus of Kra. Int. J. Primatol. 23, 161-178.

Tosi, A.J., Morales, J.C., Melnick, D.J., 2003. Paternal, maternal, and biparental molecular markers provide unique windows onto the evolutionary history of macaque monkeys. Evolution 57, $1419-1435$.

Wallman, J., Hoelzer, G.A., Melnick, D.J., 1996. The effects of social structure, geographical structure, and population size on the evolution of mitochondrial DNA. 1. A simulation model. Comput. Appl. Biosci. 12, 481-489.

Wright, S., 1943. Isolation by distance. Genetics 28, 114-138.

Zhang, Y.P., Shi, L.M., 1993. Phylogenetic relationships of macaques as inferred from restriction endonuclease analysis of mitochondrial DNA. Folia Primatol. 60, 7-17. 